\title{
Joint MS-GSC Combining and Down-Link Multiuser Diversity Scheduling
}

\author{
Slim Ben Halima, Mohamed-Slim Alouini, and Khalid A. Qaraqe
}

\begin{abstract}
Exploiting multi-user diversity is one solution to efficiently use the wireless medium. The basic principle is to assign the resources to the user experiencing the best channel conditions based on a feedback information provided by different users. In this paper, we propose three multiuser scheduling schemes which are combinations between the switch and examine transmission scheme in the network layer and the joint minimum-selection generalized selection combining and adaptive modulation in the physical layer. As a first step, fairness of access is not taken into account in the design of the proposed scheduling schemes. Then, in a second step, the proposed schemes are modified in order to enable them to achieve a certain short term fairness of access. In both cases, we study the performance of the proposed schemes in terms of spectral efficiency, feedback load and rate, and average number of combined and estimated paths. Using some selected numerical examples, the proposed schemes are compared among themselves and also to some recently published schemes.
\end{abstract}

Index Terms-Multi-user diversity, adaptive modulation, adaptive combining.

\section{INTRODUCTION}

$\mathbf{F}$ UTURE wireless communication systems must provide high data rates to respond to the growing demands of multimedia services. On the other hand, the wireless channel capacity is a scarce resource that must be used efficiently to respond to these desired requirements. Assuming a large number of active users in a cell, one way to efficiently use this resource, thus extracting a certain diversity gain, is optimum scheduling between these multiple users experiencing different channel conditions. In fact, in conventional communications systems (GSM for example), time is divided into fixed size time slots and each of these time slots is assigned to a fixed user regardless of its channel condition. However, it is highly probable that at any given point in time, at least one of the other users has a better channel quality. Multi-user diversity [1]-[3] gain can be achieved by scheduling the user having the best channel conditions. In order to manage priority between users, channel quality information (CQI) must be fed back to the base station through a dedicated uplink channel. Such information must be signalled regularly in order to respond to channel time variation.

Manuscript received June 11, 2007; revised November 27, 2008; accepted March 25, 2009. The associate editor coordinating the review of this paper and approving it for publication was M. Chiang.

S. B. Halima is with the Tunisia Polytechnic School, La Marsa, Tunisia.

M.-S. Alouini and K. A. Qaraqe are with the Department of Electrical Engineering, Texas A\&M University at Qatar, Education City, Doha, Qatar (e-mail: \{alouini, khalid.qaraqe\} @ qatar.tamu.edu).

This work was supported in part by the Qatar Foundation for Education, Science, and Community Development and in part by Qatar Telecom.

Digital Object Identifier 10.1109/TWC.2009.070629
The second issue to be dealt with is how the channel conditions are exploited by the system. In fact, adaptive modulation [4]-[6] and diversity combining are two important tools to respond to high data rates demands. More specifically adaptive modulation can achieve high spectral efficiency over wireless channels. The basic idea of adaptive modulation is to match the modulation parameters, such as constellation size, to fading channel conditions while maintaining the instantaneous error rate below a target value. Usually, the modulation mode is chosen based on the comparison results of received signal strength with several predetermined thresholds. On The other side, diversity combining makes higher transmission rates possible. However, in the downlink, diversity combining is constrained by the limited lifetime of the user equipment battery which has led to the adoption of adaptive diversity combining techniques [7]-[9]. Minimum-selection generalized selection combining (MS-GSC) [8] comes as one of these methods. It is based on the generalized selection combining (GSC) scheme. In particular, it selects the best $L_{c}$ paths among the $L$ available paths but stops the combining operation when reaching a predetermined target signal to noise ratio (SNR) reducing as a consequence the number of active maximum ratio combining (MRC) branches and as such the processing power consumption.

It is clear that adaptive modulation can benefit from diversity combining through the improved channel quality but the more interesting point is that both adaptive combining and adaptive modulation use predetermined thresholds in their operation. Based on this observation, we combine these two concepts and develop and analyze in this paper some joint adaptive combining and high speed data access schemes. We proposed three scheduling schemes that are combinations between the switch and examine transmission (SET) scheme [2] in the network layer and the adaptive modulation and MS-GSC combining in the physical layer: (1) the feedback efficient scheme (FBEF) aiming at minimizing the feedback load to the detriment of spectral efficiency. (2) the bandwidth efficient scheme (BWE) aiming at achieving high bandwidth efficiency without major consideration of the processing power consumption issue, and (3) the bandwidth efficient power greedy scheme aiming at achieving the highest bandwidth efficiency with the minimum processing power consumption. For these three schemes under consideration we evaluate their average spectral efficiency (quantified in terms of average number of bits $/ \mathrm{s} / \mathrm{Hz}$ ), the average feedback load (quantified in terms of the mean of the number of probed users), the average feedback rate (quantified in terms of the mean number of feedback bits per scheduling operation), and the average 
processing power consumption (quantified in terms of the average number of estimated and combined paths per scheduled user).

From another perspective, assigning the channel to the best user can lead to the monopolization of the resources by a limited number of users. Therefore, in the second part of the paper we study an algorithm that attempts to improve the fairness of access among the users. In other words, we try to ensure short-term fairness and guarantee more frequent access to all users (this has some implications on delay minimization). In our study, we evaluate the new system performance in terms of spectral efficiency and feedback load.

The remainder of this paper is organized as follows. The next section gives a general background on the channel and system models. Section III is devoted to the operation of the proposed schemes without fairness consideration. Section IV deals with the system that tries to achieve a better fairness of access between the scheduled users. Finally, the paper concludes with a summary of the main results.

\section{General BACKGROUnd}

\section{A. System Model}

We consider a base station (BS) serving $K$ users each having $L$ diversity branches. We consider a discrete time implementation for the proposed schemes. In particular, short guard periods are periodically inserted into the the transmitted signal. During these guard periods the BS probes each of the users in order to select the most appropriate one according to the adopted scheduling scheme. During the probing operation users perform a set of operations including path estimation, combined SNRs comparison, and modulation index determination followed by CQI feedback which is the basis for the scheduling scheme. After deciding the modulation mode, the transmitter and the selected receiver are configured accordingly throughout the subsequent data burst transmission.

Concerning the receiver and due to complexity and processing power constraints we assume that it can not combine more than $L_{c}$ diversity paths $\left(L_{c}<L\right)$. As such the receiver chooses the most appropriate paths to reach the desired modulation index under a certain bit error rate (BER) constraint.

\section{B. Channel Model}

To achieve multi-path diversity, we consider a frequency selective channel. The diversity paths represent the different resolvable multi-paths in wideband code division multiple access W-CDMA or ultra wide band UWB systems. We assume a block fading channel, in which, the sum of the guard period and the data burst time slot is roughly equal to the channel coherence time. Thus, during each time slot, the faded signal amplitude remains roughly constant during the data burst and the previous guard period.

Finally, signals among users are also assumed to be independent and identically distributed (i.i.d.) and the signals of the $L$ multi paths at each user end are assumed to be i.i.d. and to experience Rayleigh type of fading.

\section{Adaptive Combining}

Diversity combining requires different operations including paths estimation and signal processing of the different diversity paths. These operations will lead to extra power consumption and therefore reduce the valuable battery lifetime of mobile terminals. To remedy to such problem, different output-SNR oriented schemes [7]-[9] were developed. MSGSC is one of these schemes [8] and is based on GSC combining [10, chapter 9]. In fact, instead of using all the $L_{c}$ branches of GSC, the MS-GSC scheme uses the best paths that enable it to reach the target SNR. As a consequence, MSGSC can save a considerable amount of processing power by keeping fewer branches active while still reaching the desired diversity gain.

\section{Adaptive Modulation}

The process of adaptive modulation starts by examining the channel SNR at the receiver side. This SNR range is divided into $N+1$ fading regions, each of them is associated with a particular quadrature amplitude modulation (QAM) signal constellation. More specifically, the divided SNR regions are defined by the following SNR thresholds: $\gamma_{T}^{n} \leq \gamma<\gamma_{T}^{n+1}$ where $\gamma_{T}^{n}$ is the SNR threshold for $2^{n}$-QAM. Given these SNR thresholds and given the SNR estimated at the receiver, this receiver determines the modulation index $n$, such that $\gamma_{T}^{n} \leq$ $\gamma<\gamma_{T}^{n+1}$ and informs via the feedback path the transmitter about the modulation index $n$ to be used.

Assuming the M-QAM signalling, the SNR thresholds for a target BER can be determined using the following equation given in [4, Eq (28)]

$$
\gamma_{T}^{n}=-\frac{2}{3} \ln \left(5 \mathrm{BER}_{0}\right)\left(2^{n}-1\right) ; \quad n=1,2, \ldots, N
$$

where $B E R_{0}$ is the specified BER.

\section{E. Notations}

In the following sections, we will adopt the following notations and relations. We denote by $\Gamma_{i}=\sum_{l=1}^{i} \gamma_{l: L}$ the sum of the first $i$ ordered path SNRs $\gamma_{1: L} \geq \gamma_{2: L} \geq \ldots \geq \gamma_{i: L}$ and its statistics are given in [10, chapter 9]. We also denote by $\Gamma_{i}^{k}$ the sum of the first $i$ ordered path SNRs for the user $k$. The joint probability density function (PDF) of $\Gamma_{i}, \Gamma_{i-1}$ and $\Gamma_{L_{c}}$ can be shown to be given by

$$
\begin{aligned}
p_{\Gamma_{i}, \Gamma_{i-1}, \Gamma_{L_{c}}}\left(\gamma_{i}, \gamma_{i-1}, \gamma_{L_{c}}\right)= & p_{\Gamma_{i-1}, \gamma_{i: L}, \Gamma_{i}^{+}}\left(\gamma_{i-1}, \gamma_{i}-\gamma_{i-1}, \gamma_{L_{c}}-\gamma_{i}\right) \\
& \times U\left(\gamma_{L_{c}}-\gamma_{i}\right) U\left(\gamma_{i}-\gamma_{i-1}\right) \\
& \times U\left(\gamma_{i}+\left(L_{c}-i\right)\left(\gamma_{i}-\gamma_{i-1}\right)-\gamma_{L_{c}}\right) \\
& \times U\left(\frac{i}{i-1} \gamma_{i-1}-\gamma_{i}\right)
\end{aligned}
$$

where $U($.$) is the unit step function and \Gamma_{i}^{+}=\sum_{l=i+1}^{L c} \gamma_{l: L}$. Finally, the joint PDF of $\Gamma_{i-1}, \gamma_{i: L}$ and $\Gamma_{i}^{+}, p_{\Gamma_{i-1}, \gamma_{i: L}, \Gamma_{i}^{+}}$, is given in [11, Eq (21)] for $L_{c}=L$ and in [12, Eq (9)] for $L_{c}<L$. 


\section{Multiuser SCHEduling Without FAIRnESS OF ACCESS CONSIDERATION}

\section{A. Feedback Efficient Scheme}

1) Mode of Operation: During the guard period, the BS starts by randomly probing one user among the $K$ users. This user runs the MS-GSC algorithm in order to determine the maximum constellation size it can reach with respect to the target BER. More specifically, at the user level, we operate as follows. First, we set $\gamma_{T}^{N}$ as the threshold SNR. If this threshold can be reached, the receiver sets to $N$ the modulation index. Otherwise, it reduces the modulation index and repeats the previous operation. This process continues until the user finds an acceptable modulation index or it fails to reach the SNR threshold corresponding to the binary modulation.

In the first case, the user feeds back $b_{1}=\left\lfloor\log _{2}(N)\right\rfloor+1$ bits indicating the modulation index, where $\lfloor$.$\rfloor is the floor$ operator. While in the latter case, it feeds back only one negative acknowledge (NACK) bit.

At the BS level, if a positive response is received from a particular user, this user with the corresponding modulation index is serviced. Otherwise, it probes another user with the same procedure described before. Finally, if the BS does not find an acceptable user among the $K$ active users, it buffers the data.

2) Performance Analysis:

- Average spectral efficiency:

The starting point for the computation of the average spectral efficiency, $\bar{\eta}$, is the following relations

$$
\begin{aligned}
\operatorname{Pr}[\eta=n]= & \operatorname{Pr}\left[\gamma_{T}^{n} \leq \Gamma_{L_{C}}^{1}<\gamma_{T}^{n+1}\right. \\
& \text { or } \ldots \text { or } \Gamma_{L_{C}}^{1}<\gamma_{T}^{1} ; \ldots ; \Gamma_{L_{C}}^{K-1}<\gamma_{T}^{1} \\
& \left.\& \gamma_{T}^{n} \leq \Gamma_{L_{C}}^{K}<\gamma_{T}^{n+1}\right], \quad n=1, \ldots, N-1 ; \\
\operatorname{Pr}[\eta=N]= & \operatorname{Pr}\left[\Gamma_{L_{C}}^{1} \geq \gamma_{T}^{N} \text { or } \Gamma_{L_{C}}^{1}<\gamma_{T}^{1} ; \Gamma_{L_{C}}^{2} \geq \gamma_{T}^{N}\right. \\
& \text { or } \ldots \text { or } \Gamma_{L_{C}}^{1}<\gamma_{T}^{1} ; \ldots ; \Gamma_{L_{C}}^{K-1}<\gamma_{T}^{1} \\
& \left.\& \Gamma_{L_{C}}^{K} \geq \gamma_{T}^{N}\right] .
\end{aligned}
$$

Considering the fact that the events are mutually exclusive and that the users are experiencing independent channel conditions, we can rewrite (3) as follows

$$
\begin{aligned}
\operatorname{Pr}[\eta=n]= & \sum_{k=0}^{K-1}\left(P_{\Gamma_{L_{c}}}\left(\gamma_{T}^{1}\right)\right)^{k} \\
& \times\left(P_{\Gamma_{L_{c}}}\left(\gamma_{T}^{n+1}\right)-P_{\Gamma_{L_{c}}}\left(\gamma_{T}^{n}\right)\right), n=1, \ldots, N-1 ; \\
\operatorname{Pr}[\eta=N]= & \left(1-P_{\Gamma_{L_{c}}}\left(\gamma_{T}^{N}\right)\right) \sum_{k=0}^{K-1}\left(P_{\Gamma_{L_{c}}}\left(\gamma_{T}^{1}\right)\right)^{k} .
\end{aligned}
$$

Finally, the expression of the average spectral efficiency, $\bar{\eta}$, is given by

$$
\begin{aligned}
\bar{\eta}= & \sum_{n=1}^{N-1} \sum_{k=0}^{K-1} n\left(P_{\Gamma_{L_{c}}}\left(\gamma_{T}^{1}\right)\right)^{k}\left(P_{\Gamma_{L_{c}}}\left(\gamma_{T}^{n+1}\right)-P_{\Gamma_{L_{c}}}\left(\gamma_{T}^{n}\right)\right) \\
& +N\left(1-P_{\Gamma_{L_{c}}}\left(\gamma_{T}^{N}\right)\right) \sum_{k=0}^{K-1}\left(P_{\Gamma_{L_{c}}}\left(\gamma_{T}^{1}\right)\right)^{k}
\end{aligned}
$$

where $P_{\Gamma_{L_{c}}}($.$) is the cumulative distribution function (CDF)$ of $\Gamma_{L_{c}}$ and which is given by [10, chapter 9].

\section{- Average feedback load:}

The feedback load denotes the number of users giving feedback in a scheduling operation.

The computation of the probabilities for the feedback load, $F$, is given by these relations

$$
\begin{aligned}
\operatorname{Pr}[F=1]= & \operatorname{Pr}\left[\Gamma_{L_{C}}^{1} \geq \gamma_{T}^{1}\right], \\
\operatorname{Pr}[F=k]= & \operatorname{Pr}\left[\Gamma_{L_{C}}^{1}<\gamma_{T}^{1} ; \ldots ; \Gamma_{L_{C}}^{k-1}<\gamma_{T}^{1}\right. \\
& \left.\& \Gamma_{L_{C}}^{k} \geq \gamma_{T}^{1}\right], \quad k=2, \ldots, K-1 ; \\
\operatorname{Pr}[F=K]= & \operatorname{Pr}\left[\Gamma_{L_{C}}^{1}<\gamma_{T}^{1} ; \ldots ; \Gamma_{L_{C}}^{K-1}<\gamma_{T}^{1}\right] .
\end{aligned}
$$

Taking into account the independence between the random variables, we can rewrite these expressions as

$$
\begin{aligned}
\operatorname{Pr}[F=k]= & \left(1-P_{\Gamma_{L_{c}}}\left(\gamma_{T}^{1}\right)\right) \\
& \times\left(P_{\Gamma_{L_{c}}}\left(\gamma_{T}^{1}\right)\right)^{k-1}, \quad k=1, \ldots,(K-1) ; \quad(7) \\
\operatorname{Pr}[F=K]= & \left(1-P_{\Gamma_{L_{c}}}\left(\gamma_{T}^{1}\right)\right)\left(P_{\Gamma_{L_{c}}}\left(\gamma_{T}^{1}\right)\right)^{K-1}+\left(P_{\Gamma_{L_{c}}}\left(\gamma_{T}^{1}\right)\right)^{K} .
\end{aligned}
$$

The final expression of the average feedback load, $\bar{F}$, is therefore given by

$$
\bar{F}=\sum_{k=1}^{K} k\left(1-P_{\Gamma_{L_{c}}}\left(\gamma_{T}^{1}\right)\right)\left(P_{\Gamma_{L_{c}}}\left(\gamma_{T}^{1}\right)\right)^{k-1}+K\left(P_{\Gamma_{L_{c}}}\left(\gamma_{T}^{1}\right)\right)^{K}
$$

- Average feedback rate per scheduling operation:

The feedback rate per scheduling operation denotes the number of fed back bits.

In this section, we consider that the modulation indexes are coded in $b_{1}$ bits. Thus, the random variable relative to the average feedback rate per scheduling operation takes the values $K$ or $\left\{b_{1}+k\right.$ for $\left.k=0, \ldots, K-1\right\}$. On the other hand, we have

$$
\begin{array}{ll}
\operatorname{Pr}[R=K] & =\operatorname{Pr}\left[\Gamma_{L_{C}}^{1}<\gamma_{T}^{1} ; \ldots ; \Gamma_{L_{C}}^{K}<\gamma_{T}^{1}\right], \\
\operatorname{Pr}\left[R=b_{1}+k\right]= & \operatorname{Pr}[F=k+1], \quad k=0, \ldots, K-2 ; \\
\operatorname{Pr}\left[R=b_{1}+K-1\right]= & \operatorname{Pr}\left[\Gamma_{L_{C}}^{N}<\gamma_{T}^{1} ; \ldots ; \Gamma_{L_{C}}^{K-1}<\gamma_{T}^{1}\right. \\
& \left.\& \Gamma_{L_{C}}^{K} \geq \gamma_{T}^{1}\right] .
\end{array}
$$

Consequently, the average feedback rate per scheduling operation, $\bar{R}$, is

$$
\begin{aligned}
\bar{R}= & \sum_{k=0}^{K-1}\left(b_{1}+k\right)\left(1-P_{\Gamma_{L_{c}}}\left(\gamma_{T}^{1}\right)\right)\left(P_{\Gamma_{L_{c}}}\left(\gamma_{T}^{1}\right)\right)^{k} \\
& +K\left(P_{\Gamma_{L_{c}}}\left(\gamma_{T}^{1}\right)\right)^{K} .
\end{aligned}
$$

- Average number of combined paths per scheduled user:

The average number of combined paths per scheduled user, $\bar{N}_{c}$, is given by

$$
\bar{N}_{C}=\sum_{l=1}^{L_{c}} l \operatorname{Pr}\left[N_{c}=l\right],
$$

where

$$
\operatorname{Pr}\left[N_{c}=l\right]=\sum_{k=1}^{K} \operatorname{Pr}\left[\text { user } k \text { selected and } N_{c}=l\right],
$$

and

$\operatorname{Pr}\left[\right.$ user $k$ selected and $\left.N_{c}=l\right]=\sum_{n=1}^{N} \operatorname{Pr}[$ user $k$ selected $\& \eta=n$ $\left.\& N_{c}=l\right]$.

These probabilities are calculated in what follows 


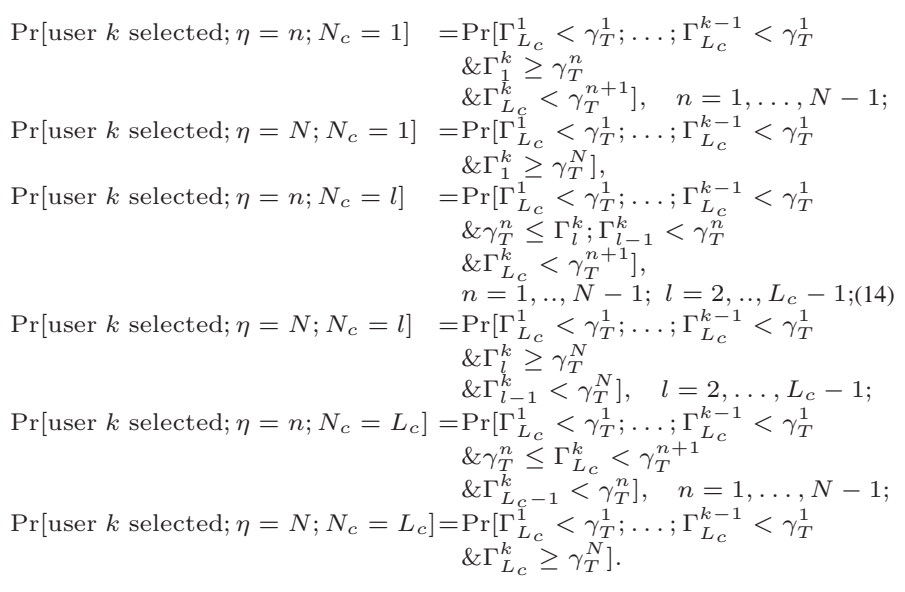

Based on the fact that the users are experiencing i.i.d. channel conditions, we have

$$
\begin{aligned}
& \operatorname{Pr}\left[N_{c}=1\right]=\sum_{k=0}^{K-1}\left(P_{\Gamma_{L_{c}}}\left(\gamma_{T}^{1}\right)\right)^{k} \\
& \times\left[\sum_{n=1}^{N-1} \int_{0}^{\infty} \int_{\gamma_{T}^{n}}^{\infty} \int_{0}^{\gamma_{T}^{n+1}} p_{\Gamma_{2}, \Gamma_{1}, \Gamma_{L_{c}}}(x, y, z) d x d y d z\right. \\
& \left.+\left(1-P_{\Gamma_{1}}\left(\gamma_{T}^{N}\right)\right)\right] \text {, } \\
& \operatorname{Pr}\left[N_{c}=l\right]=\sum_{k=0}^{K-1}\left(P_{\Gamma_{L_{c}}}\left(\gamma_{T}^{1}\right)\right)^{k} \\
& \times\left[\left(\sum_{n=1}^{N-1} \int_{\gamma_{T}^{n}}^{\infty} \int_{0}^{\gamma_{T}^{n}} \int_{0}^{\gamma_{T}^{n+1}} p_{\Gamma_{i}, \Gamma_{i-1}, \Gamma_{L_{c}}}(x, y, z) d x d y d z(1\right.\right. \\
& \left.+\left(P_{\Gamma_{l-1}}\left(\gamma_{T}^{N}\right)-P_{\Gamma_{l}}\left(\gamma_{T}^{N}\right)\right)\right], \quad l=2, \ldots,\left(L_{c}-1\right) ; \\
& \operatorname{Pr}\left[N_{c}=L_{c}\right]=\sum_{k=0}^{K-1}\left(P_{\Gamma_{L_{c}}}\left(\gamma_{T}^{1}\right)\right)^{k}
\end{aligned}
$$

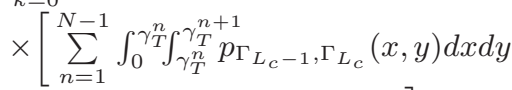

$$
\begin{aligned}
& \left.+\left(P_{\Gamma_{L_{c}-1}}\left(\gamma_{T}^{N}\right)-P_{\Gamma_{L_{c}}}\left(\gamma_{T}^{N}\right)\right)\right] \text {. }
\end{aligned}
$$

- Average number of estimated paths per user:

From the mode of operation of the MS-GSC and the proposed scheme, it is clear that the average number of estimated paths per user, $\bar{N}_{E}$, can be written as

$$
\bar{N}_{E}=\frac{L}{K} \bar{F}
$$

\section{B. Bandwidth Efficient Scheme}

1) Mode of Operation: During the guard period, the BS starts by randomly probing one user among the $K$ users. This user runs the MS-GSC algorithm setting $\gamma_{T}^{N}$ as the threshold SNR which is the SNR enabling to reach the maximum constellation size under a certain BER constraint.

If this user succeeds in providing the maximum constellation size, it feeds back one bit corresponding to a positive acknowledge (ACK) and thus it will be chosen as the served user during the next data burst. Otherwise, it feeds back $b_{2}=\left\lfloor\log _{2}(N-1)\right\rfloor+1$ bits informing the $\mathrm{BS}$ of the maximum constellation size it can reach after combining all the GSC $L_{c}$ branches.

In the second case, the BS probes another user which performs the same operation as the previous user. This procedure continues until the BS finds a user having a combined SNR greater than the $\gamma_{T}^{N}$ or all the users fail to achieve that condition. In the latter case, the BS chooses the user with the maximum provided constellation size, and if there is an outage, it buffers the data.

\section{2) Performance Analysis:}

- Average spectral efficiency:

The different probabilities corresponding to the different modulations indexes are given by

$$
\begin{aligned}
\operatorname{Pr}[\eta=n]= & \operatorname{Pr}\left[\Gamma_{L_{c}}^{1}<\gamma_{T}^{n+1} ; \ldots ; \Gamma_{L_{c}}^{K}<\gamma_{T}^{n+1}\right. \\
& \& \gamma_{T}^{n} \leq \Gamma_{L_{C}}^{1}<\gamma_{T}^{n+1} \operatorname{or} \Gamma_{L_{c}}^{1}<\gamma_{T}^{n+1} ; \ldots ; \Gamma_{L_{c}}^{K}<\gamma_{T}^{n+1} \\
& \& \Gamma_{L_{c}}^{1}<\gamma_{T}^{n} ; \Gamma_{L_{C}}^{2} \geq \gamma_{T}^{n} \text { or } \ldots \text { or } \Gamma_{L_{c}}^{1}<\gamma_{T}^{n+1} ; \ldots ; \Gamma_{L_{c}}^{K}<\gamma_{T}^{n+1}(17) \\
& \left.\& \Gamma_{L_{c}}^{1}<\gamma_{T}^{n} ; \ldots ; \Gamma_{L_{c}}^{K}<\gamma_{T}^{n} ; \Gamma_{L_{C}}^{K} \geq \gamma_{T}^{n}\right], \quad n=1, \ldots, N-1 ; \\
\operatorname{Pr}[\eta=N]= & \operatorname{Pr}\left[\Gamma_{L_{C}}^{1} \geq \gamma_{T}^{N} \text { or } \Gamma_{L}^{1}<\gamma_{T}^{N} ; \Gamma_{L_{C}}^{2} \geq \gamma_{T}^{N}\right. \\
& \text { or } \left.\ldots \text { or } \Gamma_{L_{C}}^{1}<\gamma_{T}^{N} ; \ldots ; \Gamma_{L_{C}}^{K}<\gamma_{T}^{N} ; \Gamma_{L_{C}}^{K} \geq \gamma_{T}^{N}\right] .
\end{aligned}
$$

These relations, when considering the independence between the users and the fact that the events are mutually exclusive, can be written as

$$
\begin{aligned}
\operatorname{Pr}[\eta=n]= & \sum_{k=0}^{K-1}\left(P_{\Gamma_{L_{c}}}\left(\gamma_{T}^{n}\right)\right)^{k}\left(P_{\Gamma_{L_{c}}}\left(\gamma_{T}^{n+1}\right)\right)^{K-k-1} \\
& \times\left(P_{\Gamma_{L_{c}}}\left(\gamma_{T}^{n+1}\right)-P_{\Gamma_{L_{c}}}\left(\gamma_{T}^{n}\right)\right), n=1, \ldots, N-1 ;(18) \\
\operatorname{Pr}[\eta=N]= & \left(1-P_{\Gamma_{L_{c}}}\left(\gamma_{T}^{N}\right)\right) \sum_{k=0}^{K-1}\left(P_{\Gamma_{L_{c}}}\left(\gamma_{T}^{N}\right)\right)^{k} .
\end{aligned}
$$

Finally the average spectral efficiency, $\bar{\eta}$, for the BWE scheme can be written as

$$
\begin{aligned}
\bar{\eta}= & \sum_{n=1}^{N-1} \sum_{k=0}^{K-1} n\left(P_{\Gamma_{L_{c}}}\left(\gamma_{T}^{n}\right)\right)^{k}\left(P_{\Gamma_{L_{c}}}\left(\gamma_{T}^{n+1}\right)\right)^{K-k-1} \\
& \times\left(P_{\Gamma_{L_{c}}}\left(\gamma_{T}^{n+1}\right)-P_{\Gamma_{L_{c}}}\left(\gamma_{T}^{n}\right)\right) \\
& +N\left(1-P_{\Gamma_{L_{c}}}\left(\gamma_{T}^{N}\right)\right) \sum_{k=0}^{K-1}\left(P_{\Gamma_{L_{c}}}\left(\gamma_{T}^{N}\right)\right)^{k}
\end{aligned}
$$

- Average feedback load:

The starting point to obtain the average feedback load, $\bar{F}$, expression is the following relations

$$
\begin{aligned}
\operatorname{Pr}[F=1]= & \operatorname{Pr}\left[\Gamma_{L_{C}}^{1} \geq \gamma_{T}^{N}\right], \\
\operatorname{Pr}[F=k]= & \operatorname{Pr}\left[\Gamma_{L_{C}}^{1}<\gamma_{T}^{N} ; \ldots ; \Gamma_{L_{C}}^{k-1}<\gamma_{T}^{N}\right. \\
& \left.\& \Gamma_{L_{C}}^{k} \geq \gamma_{T}^{N}\right], \quad k=2, \ldots, K-1 ; \\
\operatorname{Pr}[F=K]= & \operatorname{Pr}\left[\Gamma_{L_{C}}^{1}<\gamma_{T}^{N} ; \ldots ; \Gamma_{L_{C}}^{K-1}<\gamma_{T}^{N}\right] .
\end{aligned}
$$

These relations can be rewritten as

$$
\begin{aligned}
\operatorname{Pr}[F=1]= & 1-P_{\Gamma_{L_{C}}}\left(\gamma_{T}^{N}\right), \\
\operatorname{Pr}[F=k]= & \left(1-P_{\Gamma_{L_{c}}}\left(\gamma_{T}^{N}\right)\right) \\
& \times\left(P_{\Gamma_{L_{c}}}\left(\gamma_{T}^{N}\right)\right)^{k-1}, \quad k=2, \ldots, K-1 ; \\
\operatorname{Pr}[F=K]= & \left(1-P_{\Gamma_{L_{c}}}\left(\gamma_{T}^{N}\right)\right)\left(P_{\Gamma_{L_{c}}}\left(\gamma_{T}^{N}\right)\right)^{K-1}+\left(P_{\Gamma_{L_{c}}}\left(\gamma_{T}^{N}\right)\right)^{K} .
\end{aligned}
$$

As a consequence, the final average feedback load, $\bar{F}$, can be written as

$$
\begin{aligned}
\bar{F}= & \sum_{k=1}^{K} k\left(1-P_{\Gamma_{L_{c}}}\left(\gamma_{T}^{N}\right)\right)\left(P_{\Gamma_{L_{c}}}\left(\gamma_{T}^{N}\right)\right)^{k-1} \\
& +K\left(P_{\Gamma_{L_{c}}}\left(\gamma_{T}^{N}\right)\right)^{K} .
\end{aligned}
$$

- Average feedback rate per scheduling operation:

As in the FBEF scheme, we define $b_{2}$ as the number of bits necessary to code the modulation indices. In this case, the average feedback rate per probing operation takes the values $K b_{2}$ or $\left\{k b_{2}+1\right.$ for $k=0, \ldots, K-1\}$. The probabilities associated with the feedback rate can be written as

$$
\begin{array}{ll}
\operatorname{Pr}\left[R=K b_{2}\right] & \operatorname{Pr}\left[\Gamma_{L_{C}}^{1}<\gamma_{T}^{N} ; \ldots ; \Gamma_{L_{C}}^{K}<\gamma_{T}^{N}\right], \\
\operatorname{Pr}\left[R=k b_{2}+1\right] & \operatorname{Pr}[F=k+1], \quad k=0, \ldots, K-2 ; \\
\operatorname{Pr}\left[R=(K-1) b_{2}+1\right]= & \operatorname{Pr}\left[\Gamma_{L_{C}}^{N}<\gamma_{T}^{N} ; \ldots ; \Gamma_{L_{C}}^{K-1}<\gamma_{T}^{N}\right. \\
& \left.\& \Gamma_{L_{C}}^{K} \geq \gamma_{T}^{N}\right] .
\end{array}
$$


Finally, the average feedback rate per scheduling operation can be written as

$$
\begin{aligned}
\bar{R}= & \sum_{k=0}^{K-1}\left(k b_{2}+1\right)\left(1-P_{\Gamma_{L_{c}}}\left(\gamma_{T}^{N}\right)\right)\left(P_{\Gamma_{L_{c}}}\left(\gamma_{T}^{N}\right)\right)^{k} \\
& +K b_{2}\left(P_{\Gamma_{L_{c}}}\left(\gamma_{T}^{N}\right)\right)^{K} .
\end{aligned}
$$

- Average number of combined paths per scheduled user:

The average number of combined paths per scheduled user, $\bar{N}_{c}$, is given by

$$
\bar{N}_{C}=\sum_{l=1}^{L_{c}} l \operatorname{Pr}\left[N_{c}=l\right]
$$

where

$$
\operatorname{Pr}\left[N_{c}=l\right]=\sum_{k=1}^{K} \operatorname{Pr}\left[\text { user } k \text { is selected } \& N_{c}=l\right], l=1, ., L_{c}-1 ;(26)
$$

which is given by

$$
\begin{aligned}
\operatorname{Pr}\left[\text { user } k \text { is selected and } N_{c}=1\right]= & \operatorname{Pr}\left[\Gamma_{L_{c}}^{1}<\gamma_{T}^{N} ; \ldots ; \Gamma_{L_{c}}^{k-1}<\gamma_{T}^{N}\right. \\
& \left.\& \Gamma_{1}^{k} \geq \gamma_{T}^{N}\right], \\
\operatorname{Pr}\left[\text { user } k \text { is selected and } N_{c}=l\right]= & \operatorname{Pr}\left[\Gamma_{L_{c}}^{1}<\gamma_{T}^{N} ; \ldots ; \Gamma_{L_{c}}^{k-1}<\gamma_{T}^{N}(27)\right. \\
& \& \gamma_{T}^{N} \leq \Gamma_{l}^{k} \\
& \left.\& \Gamma_{l-1}^{k}<\gamma_{T}^{N}\right], \quad l=2, \ldots, L_{c}-1 ;
\end{aligned}
$$

and

$$
\begin{aligned}
\operatorname{Pr}\left[N_{c}=L_{c}\right]= & \operatorname{Pr}\left[\Gamma_{L_{c}}^{1} \geq \gamma_{T}^{N} ; \Gamma_{L_{c}-1}^{1}<\gamma_{T}^{N} \text { or } \Gamma_{L_{c}}^{1}<\gamma_{T}^{N}\right. \\
& \& \Gamma_{L_{c}}^{2} \geq \gamma_{T}^{N} ; \Gamma_{L_{c}-1}^{2}<\gamma_{T}^{N} \\
& \text { or } \ldots \text { or } \Gamma_{L_{c}}^{1}<\gamma_{T}^{N} ; \ldots ; \Gamma_{L_{c}}^{K-1}<\gamma_{T}^{N} \\
& \& \Gamma_{L_{c}}^{K} \geq \gamma_{T}^{N} ; \Gamma_{L_{c}-1}^{K}<\gamma_{T}^{N} \\
& \text { or }\left\{\Gamma_{L_{c}}^{1}<\gamma_{T}^{N} ; \ldots ; \Gamma_{L_{c}}^{K}<\gamma_{T}^{N}\right. \\
& \&\left(\gamma_{T}^{1} \leq \Gamma_{L_{c}}^{1}<\gamma_{T}^{N} \text { or } \Gamma_{L_{c}}^{1}<\gamma_{T}^{1} ; \gamma_{T}^{1} \leq \Gamma_{L_{c}}^{2}<\gamma_{T}^{N}\right. \\
& \text { or } \left.\left.\left.\ldots \text { or } \Gamma_{L_{c}}^{1}<\gamma_{T}^{1} ; \ldots ; \Gamma_{L_{c}}^{K-1}<\gamma_{T}^{1} ; \gamma_{T}^{1} \leq \Gamma_{L_{c}}^{K}<\gamma_{T}^{N}\right)\right\}\right] .
\end{aligned}
$$

As a consequence, we have

$$
\begin{aligned}
\operatorname{Pr}\left[N_{c}=1\right]= & \sum_{k=0}^{K-1}\left(P_{\Gamma_{L_{c}}}\left(\gamma_{T}^{N}\right)\right)^{k}\left(1-P_{\Gamma_{1}}\left(\gamma_{T}^{N}\right)\right), \\
\operatorname{Pr}\left[N_{c}=l\right]= & \sum_{k=0}^{K-1}\left(P_{\Gamma_{L_{c}}}\left(\gamma_{T}^{N}\right)\right)^{k} \\
& \times\left(P_{\Gamma_{l-1}}\left(\gamma_{T}^{N}\right)-P_{\Gamma_{l}}\left(\gamma_{T}^{N}\right)\right), \quad l=1, \ldots, L_{c} ;(29) \\
\operatorname{Pr}\left[N_{c}=L_{c}\right]= & \sum_{k=0}^{K-1}\left(P_{\Gamma_{L_{c}}}\left(\gamma_{T}^{N}\right)\right)^{k}\left(P_{\Gamma_{L_{c}-1}}\left(\gamma_{T}^{N}\right)-P_{\Gamma_{L_{c}}}\left(\gamma_{T}^{N}\right)\right) \\
& +\sum_{k=0}^{K-1}\left(P_{\Gamma_{L_{c}}}\left(\gamma_{T}^{1}\right)\right)^{k}\left(P_{\Gamma_{L_{c}}}\left(\gamma_{T}^{N}\right)\right)^{K-k-1} \\
& \times\left(P_{\Gamma_{L_{c}}}\left(\gamma_{T}^{N}\right)-P_{\Gamma_{L_{c}}}\left(\gamma_{T}^{1}\right)\right) .
\end{aligned}
$$

- Average number of estimated paths per user:

Similar to the previous section, we can write the average number of estimated paths per user, $\bar{N}_{E}$, as follows

$$
\bar{N}_{E}=\frac{L}{K} \bar{F}
$$

\section{Bandwidth Efficient Power Greedy Scheme}

1) Mode of Operation: In a first step, the BS probes the users for the highest modulation index. In the beginning, the BS starts by randomly probing one user among the $K$ users. This user runs the MS-GSC algorithm setting $\gamma_{T}^{N}$ as the threshold SNR.

If this user succeeds in providing the maximum constellation size, it feeds back one bit corresponding to an ACK and it is served by the BS in the next data burst. Otherwise, it feeds back one bit corresponding to a NACK. In the latter case, the BS probes another user which runs also the MS-GSC algorithm with the threshold corresponding to the maximum constellation size and then feeds back the appropriate acknowledgement. This operation continues until the BS finds a user having a combined SNR greater than $\gamma_{T}^{N}$ or all the users fail to achieve that condition. In the latter case the BS reduces the constellation size (i.e. it sets $\gamma_{T}^{N-1}$ as the new threshold SNR in our case) and repeats the probing operation as described before for this newly set threshold. The BS continues probing users until an acceptable one with appropriate channel conditions is selected. In the case of an outage, the BS buffers the data.

\section{2) Performance Analysis:}

- Average spectral efficiency:

Based on the mode of operation of this scheme, its average spectral efficiency is exactly the same as the average spectral efficiency of the BWE scheme.

\section{- Average feedback load:}

As for the average spectral efficiency, the average feedback load for this scheme is given by the same relation as the BWE scheme.

- Average feedback rate per scheduling operation:

Based on the mode of operation of this scheme, the random variable corresponding to the number of bits per scheduling operation takes the values $\{n K+k, \quad n=1, \ldots, N-1 ; k=1, \ldots, K\}$. The different probabilities associated with the feedback rate are given by

$$
\begin{aligned}
\operatorname{Pr}[R=k]= & \operatorname{Pr}\left[\Gamma_{L_{c}}^{1}<\gamma_{T}^{N} ; \ldots ; \Gamma_{L_{c}}^{k-1}<\gamma_{T}^{N} ; \Gamma_{L_{c}}^{k} \geq \gamma_{T}^{N}\right], \\
\operatorname{Pr}[R=n K+k]= & \operatorname{Pr}\left[\Gamma_{L_{c}}^{1}<\gamma_{T}^{N-n+1} ; \ldots ; \Gamma_{L_{c}}^{K}<\gamma_{T}^{N-n+1}\right. \\
& \& \Gamma_{L_{c}}^{1}<\gamma_{T}^{N-n} ; \ldots ; \Gamma_{L_{c}}^{k-1}<\gamma_{T}^{N-n} \\
& \left.\& \Gamma_{L_{c}}^{k} \geq \gamma_{T}^{N-n}\right], \quad n=1, . ., N-1 ; k=1, . ., K-1 ; \\
= & \operatorname{Pr}\left[\Gamma_{L_{c}}^{1}<\gamma_{T}^{1} ; \ldots ; \Gamma_{L_{c}}^{K-1}<\gamma_{T}^{1} ; \gamma_{T}^{1} \leq \Gamma_{L_{c}}^{K}<\gamma_{T}^{2}\right. \\
& \left.\operatorname{or} \Gamma_{L_{c}}^{1}<\gamma_{T}^{1} ; \ldots ; \Gamma_{L_{c}}^{K}<\gamma_{T}^{1}\right] .
\end{aligned}
$$

Considering the fact that the different random variables are i.i.d., we have

$$
\begin{aligned}
\operatorname{Pr}[R=k]= & P_{\Gamma_{L_{c}}}\left(\gamma_{T}^{N}\right)^{(k-1)}\left(1-P_{\Gamma_{L_{c}}}\left(\gamma_{T}^{N}\right)\right), \\
\operatorname{Pr}[R=n K+k]= & P_{\Gamma_{L_{c}}}\left(\gamma_{T}^{N-n+1}\right)^{(K-k)} P_{\Gamma_{L_{c}}}\left(\gamma_{T}^{N-n}\right)(k-1) \\
& \times\left(P_{\Gamma_{L_{c}}}\left(\gamma_{T}^{N-n+1}\right)-P_{\Gamma_{L_{c}}}\left(\gamma_{T}^{N-n}\right)\right), \\
& n=1, \ldots, N-1 ; k=1, \ldots, K-1 ; \\
\operatorname{Pr}[R=N K]= & P_{\Gamma_{L_{c}}}\left(\gamma_{T}^{1}\right)^{(K-1)}\left(P_{\Gamma_{L_{c}}}\left(\gamma_{T}^{2}\right)-P_{\Gamma_{L_{c}}}\left(\gamma_{T}^{1}\right)\right) \\
& +P_{\Gamma_{L_{c}}}\left(\gamma_{T}^{1}\right)^{K} .
\end{aligned}
$$

and the resulting average feedback rate is thus

$$
\bar{R}=\sum_{n=0}^{N-1} \sum_{k=1}^{K}(n K+k) \operatorname{Pr}[R=n K+k] .
$$

- Average number of combined paths per scheduled user:

The average number of combined paths per scheduled user, $\bar{N}_{c}$, is given by

$$
\bar{N}_{C}=\sum_{l=1}^{L_{c}} l \operatorname{Pr}\left[N_{c}=l\right],
$$

where

$$
\operatorname{Pr}\left[N_{c}=l\right]=\sum_{n=1}^{N} \operatorname{Pr}\left[\eta=n ; N_{c}=l\right], \quad l=1, \ldots, L_{c}
$$

with

$\operatorname{Pr}\left[\eta=n ; N_{c}=l\right]=\sum_{k=1}^{K} \operatorname{Pr}\left[\right.$ user $k$ selected $\left.\& \eta=n \& N_{c}=l\right]$,

where 


$$
\begin{aligned}
\operatorname{Pr}\left[\text { user } k \text { selected \& } \eta=n ; N_{c}=1\right]= & \operatorname{Pr}\left[\Gamma_{L_{c}}^{1}<\gamma_{T}^{n+1} ; \ldots ; \Gamma_{L_{c}}^{K}<\gamma_{T}^{n+1}\right. \\
& \& \Gamma_{L_{c}}^{1}<\gamma_{T}^{n} ; \ldots ; \Gamma_{L_{c}}^{k-1}<\gamma_{T}^{n} \\
& \& \Gamma_{1}^{k} \geq \gamma_{T}^{n} \\
& \left.\& \Gamma_{L_{c}}^{k}<\gamma_{T}^{n+1}\right], n=1, \ldots, N-1 ; \\
= & \operatorname{Pr}\left[\Gamma_{L_{c}}^{1}<\gamma_{T}^{N} ; \ldots ; \Gamma_{L_{c}}^{k-1}<\gamma_{T}^{N}\right. \\
& \left.\& \Gamma_{1} \geq \gamma_{T}^{N}\right], \\
= & \operatorname{Pr}\left[\Gamma_{L_{c}}^{1}<\gamma_{T}^{n+1} ; \ldots ; \Gamma_{L_{c}}^{K}<\gamma_{T}^{n+1}\right. \\
& \& \Gamma_{L_{c}}^{1}<\gamma_{T}^{n} ; \ldots ; \Gamma_{L_{c}}^{k-1}<\gamma_{T}^{n} \\
& \& \Gamma_{l-1}^{k} \geq \gamma_{T}^{n} ; \Gamma_{l-1}^{k}<\gamma_{T}^{n} \\
& \left.\left.\& \Gamma_{L_{c}}^{k}<\gamma_{T}^{n+1}\right], n=1, \ldots, N-1 ;\right) \\
\operatorname{Pr}\left[\text { user } k \text { selected \& } \eta=n ; N_{c}=l\right] & \operatorname{Pr}\left[\Gamma_{L_{c}}^{1}<\gamma_{T}^{N} ; \ldots ; \Gamma_{L_{C}}^{k-1}<\gamma_{T}^{N}\right. \\
& \left.\& \Gamma_{l} \geq \gamma_{T}^{N} ; \Gamma_{l-1}<\gamma_{T}^{N}\right], \\
\operatorname{Pr}\left[\text { user } k \text { selected \& } \eta=N ; N_{c}=l\right] & \\
\operatorname{Pr}\left[\text { user } k \text { selected \& } \eta=n ; N_{c}=L_{c}\right]= & \operatorname{Pr}\left[\Gamma_{L_{c}}^{1}<\gamma_{T}^{n+1} ; \ldots ; \Gamma_{L_{c}}^{K}<\gamma_{T}^{n+1}\right. \\
& \& \Gamma_{L_{c}}^{1}<\gamma_{T}^{n} ; \ldots ; \Gamma_{L}^{k-1}<\gamma_{T}^{n} \\
& \& \gamma_{T}^{n} \leq \Gamma_{L_{c}}^{k}<\gamma_{T}^{n+1} \\
& \left.\& \Gamma_{L_{c}-1}^{k}<\gamma_{T}^{n}\right], n=1, \ldots, N-1 ; \\
\operatorname{Pr}\left[\text { user } k \text { selected \& } \eta=N ; N_{c}=L_{c}\right]= & \operatorname{Pr}\left[\Gamma_{L_{c}}^{1}<\gamma_{T}^{N} ; \ldots ; \Gamma_{L_{c}}^{k-1}<\gamma_{T}^{N}\right. \\
& \left.\& \Gamma_{L_{c}} \geq \gamma_{T}^{N} ; \Gamma_{L_{c}-1}<\gamma_{T}^{N}\right] .
\end{aligned}
$$

Finally, when we consider the fact that the users experience i.i.d. channel conditions, it can be shown that

$$
\begin{aligned}
& \operatorname{Pr}\left[N_{c}=1\right]=\sum_{n=1}^{N-1} \sum_{k=0}^{K-1}\left(P_{\Gamma_{L_{c}}}\left(\gamma_{T}^{n}\right)\right)^{k}\left(P_{\Gamma_{L_{c}}}\left(\gamma_{T}^{n+1}\right)\right)^{K-k-1} \\
& \times \int_{0}^{\infty} \int_{\gamma_{T}^{n} \int_{0}^{\gamma_{T}}}^{n+1} p_{\Gamma_{2}, \Gamma_{1}, \Gamma_{L_{c}}}(x, y, z) d x d y d z \\
& +\left(1-P_{\Gamma_{1}}\left(\gamma_{T}^{N}\right)\right) \sum_{k=0}^{K-1}\left(P_{\Gamma_{L_{c}}}\left(\gamma_{T}^{N}\right)\right)^{k} \\
& \operatorname{Pr}\left[N_{c}=l\right]=\sum_{n=1}^{N-1} \sum_{k=0}^{K-1}\left(P_{\Gamma_{L_{c}}}\left(\gamma_{T}^{n}\right)\right)^{k}\left(P_{\Gamma_{L_{c}}}\left(\gamma_{T}^{n+1}\right)\right)^{K-k-1} \\
& \times \int_{\gamma_{T}^{n}}^{\infty} \int_{0}^{\gamma_{T}^{n}} \int_{0}^{\gamma_{T}^{n+1}} p_{\Gamma_{i}, \Gamma_{i-1}, \Gamma_{L_{c}}}(x, y, z) d x d y d z \\
& +\sum_{k=0}^{K-1}\left(P_{\Gamma_{L_{c}}}\left(\gamma_{T}^{N}\right)\right)^{k} \\
& \times\left(P_{\Gamma_{l-1}}^{k=0}\left(\gamma_{T}^{N}\right)-P_{\Gamma_{l}}\left(\gamma_{T}^{N}\right)\right), \quad l=2, \ldots, L_{C}-1 ; \\
& \operatorname{Pr}\left[N_{c}=L_{c}\right]=\sum_{n=1}^{N-1} \sum_{k=0}^{K-1}\left(P_{\Gamma_{L_{c}}}\left(\gamma_{T}^{n}\right)\right)^{k}\left(P_{\Gamma_{L_{c}}}\left(\gamma_{T}^{n+1}\right)\right)^{K-k-1} \\
& \times \int_{0}^{\gamma_{T}^{n}} \int_{\gamma_{T}^{n}}^{\gamma_{T}^{n+1}} p_{\Gamma_{L_{c}-1}, \Gamma_{L_{c}}}(x, y) d x d y \\
& +\left(P_{\Gamma_{L_{c}-1}}\left(\gamma_{T}^{N}\right)-P_{\Gamma_{L_{c}}}\left(\gamma_{T}^{N}\right)\right) \sum_{k=0}^{K-1}\left(P_{\Gamma_{L_{c}}}\left(\gamma_{T}^{N}\right)\right)^{k} .
\end{aligned}
$$

\section{Numerical Results}

In this section, we make a comparison between the three proposed schemes as well as a comparison between these new schemes and some recently published schemes. In Fig.1, equations (5), (19), [13, Eq (17)], and [13, Eq (21)] are plotted, the MRC curve is obtained using simulation. Concerning Fig.2, equations (16), (30), [13, Eq (18)], and [13, Eq (22)] are plotted. Equations (8), and (22) are plotted in Fig.3. In Fig. 4, equations (11), (25), and (34) are represented, the MRC curve is represented using simulation. Finally, we plot in Fig. 5 equations (10), (24), and (33).

Fig. 1 shows that the spectral efficiency of the FBEF scheme is lower than the spectral efficiency of the horizontal switch and examine transmission (HSET) [13]. Fig. 2 shows that the average number of estimated paths per user for the FBEF scheme is greater than that of the vertical switch and examine transmission (VSET) [13]. In fact, according to the mode of operation of MS-GSC, it is necessary to estimate and rank all the $L$ available paths whenever we probe a new user, contrary to what is performed in the VSET which will estimate just as many paths as needed (but not necessarily combine the best ones). Fig. 1 shows also that the BWE and BEPG schemes

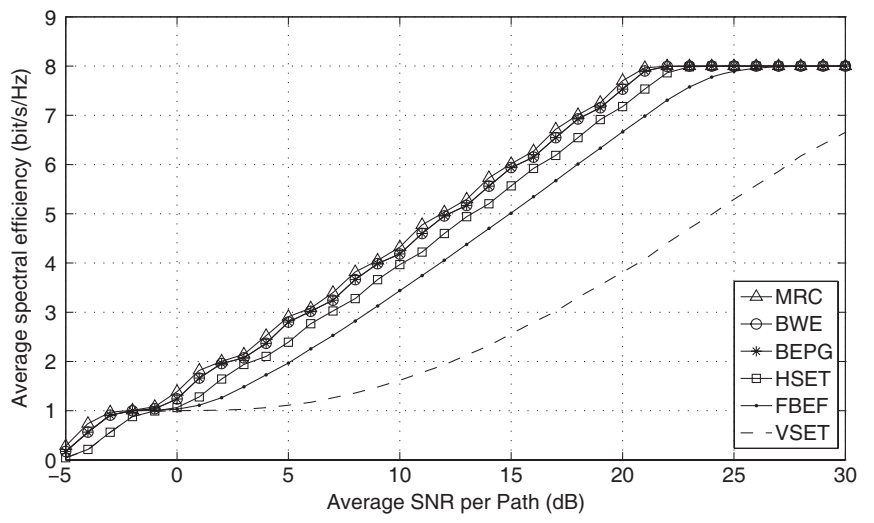

Fig. 1. Average spectral efficiency versus the average SNR for the MRC, BWE, BEPG, HSET, FBEF, and VSET schemes when $L=6, L_{c}=4, K=10$, $N=8$, and $B E R_{0}=0.001$.

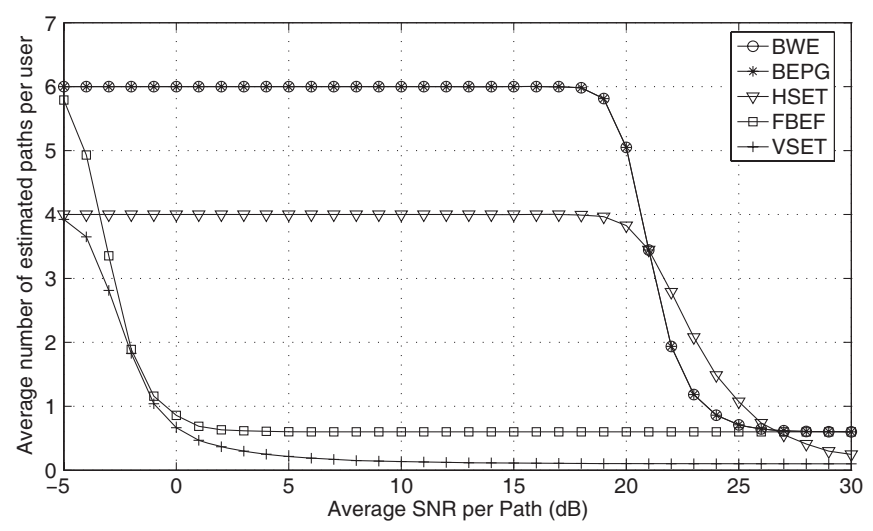

Fig. 2. Average number of estimated paths per user versus the average SNR for the BWE, BEPG, HSET, FBEF, and VSET schemes when $L=6, L_{c}=4$, $K=10, N=8$, and $B E R_{0}=0.001$.

achieve the same spectral efficiency and the best possible in comparison with HSET and FBEF. However, this comes at the expense of a high feedback load and number of estimated paths per user as an illustration in Fig. 2 and Fig. 3. Seeing that the BWE and BEPG schemes provide the maximum spectral efficiency, we also compare them with a scheme having the same mode of operation but which adopts MRC instead of MS-GSC. In other words, when a user is probed, it performs full $L$-paths MRC combining operation in order to determine the maximum spectral efficiency that can be reached. As it is shown in Fig. 1, the performance of the three schemes are not very different in terms of spectral efficiency. However, as illustrated in Fig. 4, the BWE and BEPG schemes offer considerable savings in terms of number of combined paths when compared to MRC. In addition it is clear from Fig. 4 that the BEPG scheme reduces considerably the number of combined paths compared to the BWE scheme and is as such interesting from a battery power efficiency perspective. Finally, we can see from Fig. 5 that the FBEF scheme achieves best performance from a feedback perspective but this comes at the expense of a considerable loss in terms of spectral efficiency, as illustrated in Fig. 1. Table I summarizes the key results presented in this section. 


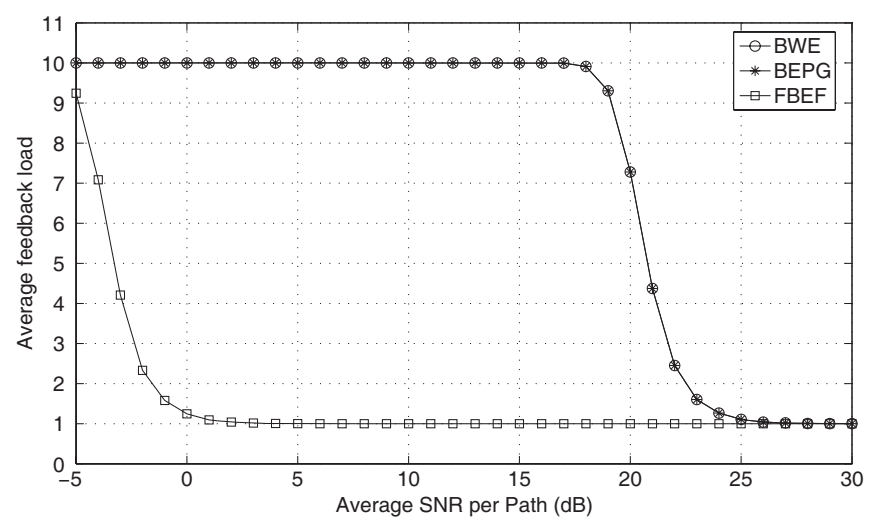

Fig. 3. Average feedback load versus the average SNR for the BWE, BEPG, and FBEF schemes when $L=6, L_{c}=4, K=10, N=8$, and $B E R_{0}=0.001$.

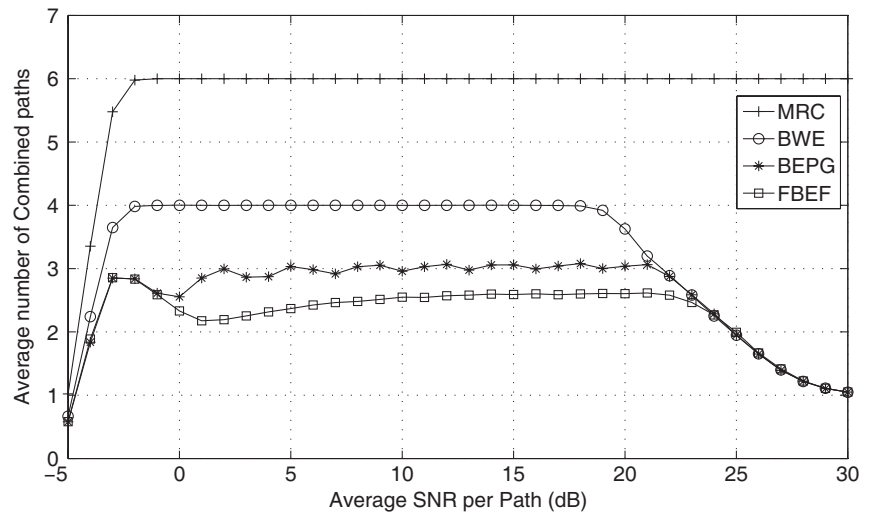

Fig. 4. Average number of combined paths per scheduled user versus the average SNR for the BWE, BEPG, FBEF, and MRC schemes when $L=6$, $L_{c}=4, K=10, N=8$, and $B E R_{0}=0.001$.

\section{Achieving Fairness between the Scheduled USERS}

\section{A. Motivation}

In the three multiuser down link scheduling schemes proposed in the previous section, the operation mode is based on probing the users in a sequential manner. More specifically,

TABLE I

SUMMARY OF THE KEY PERFORMANCE FEATURES OF THE PROPOSED SCHEMES

\begin{tabular}{|l|l|}
\hline Scheduling scheme & Key performance features \\
\hline FBEF & ○ Low spectral efficiency \\
& ○ Low feedback load \\
& ○ Low number of estimated paths \\
& ○ High power savings \\
& ○ Low feedback rate \\
\hline BWE & ○ High spectral efficiency \\
& ○ High feedback load \\
& ○ High number of estimated paths \\
& ○ Worst performance in terms of power savings \\
& ○ High feedback rate \\
\hline BEPG & ○ High spectral efficiency \\
& ○ High feedback load \\
& ○ High number of estimated paths \\
& ○ Good performance in terms of power savings \\
& ○ Highest feedback rate \\
\hline
\end{tabular}

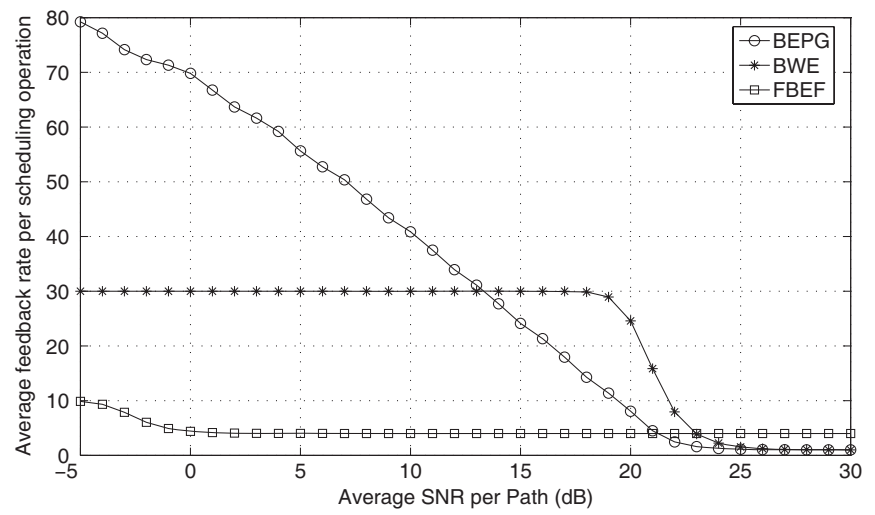

Fig. 5. Average feedback rate per scheduling operation versus the average SNR for the BWE, BEPG, and FBEF schemes when $L=6, L_{c}=4, K=10$, $N=8$, and $B E R_{0}=0.001$.

according to the feedback information, the BS selects the appropriate user having acceptable channel conditions (enabling at least BPSK transmission) which is the case for the FBEF scheme or having the best channel conditions which is the case for the BWE and BEPG schemes. Examining the mode of operation of the schemes discussed before, there is no guarantee in terms of fairness of access. In fact, with such schemes, some users may end up being deprived from service after several scheduling rounds and subjected as such to the well known starvation problem. Based on this observation, we design a scheduler that is able to ensure a certain short term fairness between the i.i.d. users, while simultaneously employing opportunistic scheduling strategies to increase the total system throughput by selecting users with high-quality channels when possible. Different fair scheduling schemes such as the proportional fair algorithm [14] were designed to achieve fairness between non i.i.d. users. In this paper, the proposed system is based on the three scheduling schemes presented in the previous section and adopts the idea of enhanced equal access (EEA) scheduling policy suggested in [15]. More specifically, in the three schemes suggested in section 3 when a user is scheduled in a time slot it is removed from the scheduling operation in the next round until all the remaining users have been scheduled. Therefore, with EEA all the users are served in the minimum possible time. However, this improvement of fairness comes at the expense of a certain degradation in the performances of the system mainly in terms of spectral efficiency. Thus, we study in the following how the performance of our proposed schemes are modified when EEA is adopted. In particular, we derive new expressions for the average spectral efficiency per time slot and the average feedback load per time slot. These results are validated using Monte-Carlo simulations.

\section{B. Mode of Operation of The Proposed Scheme}

The mode of operation of this scheme is based on the idea of EEA scheduling policy suggested in [15].To achieve the desired fairness of access between the different users the proposed scheme operates as follows:

1. In the first time slot, the BS performs the probing operation according to one of the schemes presented in section 
III on the $K$ active available users.

2. Once one user $\left(\right.$ user $\left._{1}\right)$ is selected, in the next time slot, this user is removed from the pool of the users to be scheduled and the BS performs the same previous operation on the $K-1$ remaining users until selecting another user $\left(\right.$ user $\left._{2}\right)$ among them.

3. The BS repeats the previous operations for the next time slots until the $K$ users are scheduled.

When adopting the proposed scheme we are sure to achieve an even repartition of resources between the different users. Moreover, such a scheme, and under acceptable channel conditions, guarantees a maximum inter-access time of $2 K-1$ time slots. Note that we refer by acceptable channel conditions to the fact that in each time slot of $K$ consecutive time slots we have at least one of the probed users able to reach the BPSK threshold SNR.

\section{Performance Analysis}

The scheduling process of the system is modeled as a discrete-state Markov process (Markov chain) where the number of states at each scheduling process is equal to the time slot order number $l$.

We define $\lambda(i)$ as

$$
\lambda(i)=\left(P_{\Gamma_{L_{c}}}\left(\gamma_{T}^{1}\right)\right)^{i},
$$

and we introduce the probability $\Theta(k, l)$ that in the $l^{\text {th }}$ time slot the number of users is equal to $k$ which is given in [16] by

$$
\Theta(k, l)= \begin{cases}(\lambda(K))^{l-1}, & k=K ; \\ {[(1-\lambda(k+1)) \Theta(k+1, l-1)} & \\ +\lambda(k) \Theta(k, l-1)], & (K-(l-1))<k<K ; \\ \prod_{i=0}^{l-2}(1-\lambda(K-i)), & k=K-(l-1) .\end{cases}
$$

- Average spectral efficiency per time slot:

Based on the probability that in the $l^{\text {th }}$ time slot the number of users is equal to $k$, the average spectral efficiency for the $l^{\text {th }}$ time slot can be written as

$\bar{\eta}(l)= \begin{cases}\bar{\eta}_{K}, & \text { if } l=1 ; \\ \sum_{j=1}^{l} \bar{\eta}_{K-(j-1)} \Theta(K-(j-1), l), & \text { if } 1<l \leq K\end{cases}$

where $\bar{\eta}_{k}$ is given by

$$
\bar{\eta}_{k}= \begin{cases}\sum_{n=1}^{N-1} \sum_{i=0}^{k-1} n\left(P_{\Gamma_{L_{c}}}\left(\gamma_{T}^{1}\right)\right)^{i} & \\ \times\left(P_{\Gamma_{L_{c}}}\left(\gamma_{T}^{n+1}\right)-P_{\Gamma_{L_{c}}}\left(\gamma_{T}^{n}\right)\right) & \\ +N\left(1-P_{\Gamma_{L_{c}}}\left(\gamma_{T}^{N}\right)\right) \sum_{i=0}^{k-1}\left(P_{\Gamma_{L_{c}}}\left(\gamma_{T}^{1}\right)\right)^{i}, & \text { FBEF, } \\ \sum_{n=1}^{N-1} \sum_{i=0}^{k-1} n\left(P_{\Gamma_{L_{c}}}\left(\gamma_{T}^{n}\right)\right)^{i}\left(P_{\Gamma_{L_{c}}}\left(\gamma_{T}^{n+1}\right)\right)^{k-i-1} & \\ \times\left(P_{\Gamma_{L_{c}}}\left(\gamma_{T}^{n+1}\right)-P_{\Gamma_{L_{c}}}\left(\gamma_{T}^{n}\right)\right) & \\ +N\left(1-P_{\Gamma_{L_{c}}}\left(\gamma_{T}^{N}\right)\right) \sum_{i=0}^{k-1}\left(P_{\Gamma_{L_{c}}}\left(\gamma_{T}^{N}\right)\right)^{i} . & \text { BWE/BEPG }\end{cases}
$$

Finally, the average spectral efficiency per time slot, $\bar{\eta}$, for $L_{T}$ time slots and $K$ users can be written as

$$
\bar{\eta}=\frac{1}{L_{T}} \sum_{l=1}^{L_{T}} \bar{\eta}(l)
$$

- Average feedback load per time slot:

Similarly to what was done before and based on the probability that in the $l^{\text {th }}$ time slot the number of users is equal to $k$, the average feedback load per time slot, $\bar{F}$, for $K$ users and $L_{T}$ time slots is

$$
\bar{F}=\frac{1}{L_{T}} \sum_{l=1}^{L_{T}} \bar{F}(l)
$$

where

$$
\bar{F}(l)= \begin{cases}\bar{F}_{K}, & \text { if } l=1 ; \\ \sum_{j=1}^{l} \bar{F}_{K-(j-1)} \Theta(K-(j-1), l), & \text { if } 1<l \leq K,\end{cases}
$$

and

$$
\bar{F}_{k}= \begin{cases}\sum_{i=1}^{k} i\left(1-P_{\Gamma_{L_{c}}}\left(\gamma_{T}^{1}\right)\right)\left(P_{\Gamma_{L_{c}}}\left(\gamma_{T}^{1}\right)\right)^{i-1} & \\ +k\left(P_{\Gamma_{L_{c}}}\left(\gamma_{T}^{1}\right)\right)^{k}, & \mathrm{FBEF} ; \\ \sum_{i=1}^{k} i\left(1-P_{\Gamma_{L_{c}}}\left(\gamma_{T}^{N}\right)\right)\left(P_{\Gamma_{L_{c}}}\left(\gamma_{T}^{N}\right)\right)^{i-1} & \\ +k\left(P_{\Gamma_{L_{c}}}\left(\gamma_{T}^{N}\right)\right)^{k}, & \text { BWE/BEPG. }\end{cases}
$$

- Probability that $K$ Users Have Access to the Channel in $K$ Time Slots:

The evaluation of such probability gives us an idea about the range of SNRs in which the maximum time of access between two serving operation for all the users is equal to $2 K-1$ time slots. The probability of having all the $K$ users served in $K$ time slots is given by

$$
P=\prod_{k=1}^{K}\left(1-P_{\Gamma_{L_{c}}}\left(\gamma_{T}^{1}\right)^{k}\right)
$$

\section{Numerical Results}

In this section, we present some numerical and simulation results of the new spectral efficiency and feedback load of the system. We compare the new performances of the scheduling schemes studied in section III after adoption of the EEA idea when scheduling users. Fig. 6 shows the average spectral efficiency per time slot for the system. It is clear that the cost of achieving a better fairness between the scheduled users is some loss in spectral efficiency. In fact, in the medium SNR region, the spectral efficiency per time slot is reduced considerably for the BWE and BEPG schemes but for the FBEF scheme, there is practically no loss in terms of spectral efficiency which is explained by the fact that such a scheme does not take into consideration spectral efficiency as the primary goal.

Fig. 7 illustrates the average feedback load per time slot for the system. The remark to be made is that the EEA reduces the feedback load per time slot. Indeed, Fig. 7 shows that the feedback load is reduced by almost half of its original value in the low to medium SNR regions but remains the same in the high SNR region (this is due to the fact that in these regions this value is the optimal one). Such a trend can be explained by reduction in the number of users to be scheduled as the scheduling process progresses when EEA is adopted. 


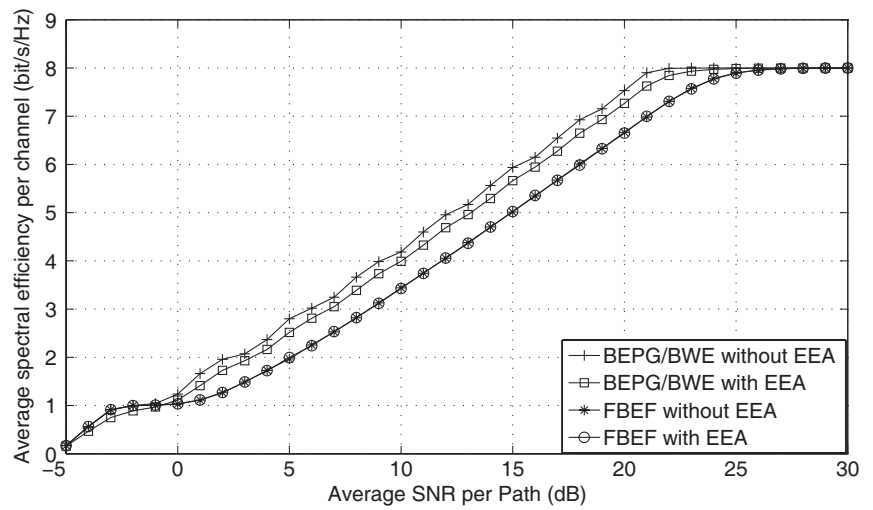

Fig. 6. Average spectral efficiency per time slot versus the average SNR for the three schemes with EEA and without EEA when $L_{T}=10, L=6$, $L_{c}=4, K=10, N=8$, and for $B E R_{0}=0.001$.

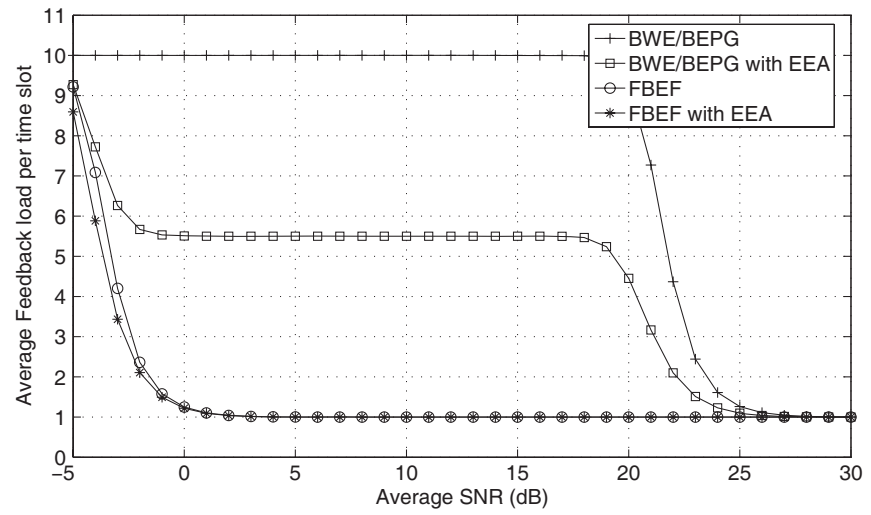

Fig. 7. Average feedback load per time slot versus the average SNR for the three schemes with EEA and without EEA when $L_{T}=10, L=6, L_{c}=4$, $K=10, N=8$, and for $B E R_{0}=0.001$.

Finally, Fig. 8 shows that starting from $5 \mathrm{~dB}$, all the $K$ users are served in $K$ time slots, when $L_{T}=K$. Such trend can be explained by the fact that in this SNR region, there is a high probability to have at least one user with an SNR higher than the BPSK threshold SNR. This also means that in this case, the maximum waiting time to have an other access to the channel is $2 K-1$ time slots. To conclude, the previous analysis shows that the performance penalties of the EEA in terms of spectral efficiency do not seem significant compared to the gain achieved in terms of feedback load and probability of fair access.

\section{CONCLUSion}

We presented in this paper three multiuser scheduling schemes based on the MS-GSC combining. We evaluated their performance and compared this performance to that of the HSET, VSET and MRC schemes. It was shown, that the FBEF achieves a minimum feedback load, while the BWE and BEPG schemes achieve the best spectral efficiency. It was also shown that there is not a significant difference between the BWE, BEPG and MRC schemes in terms of spectral efficiency. However, the two former schemes clearly outperform MRC in terms of average combined paths with a certain advantage to the BEPG scheme. The proposed schemes were used in

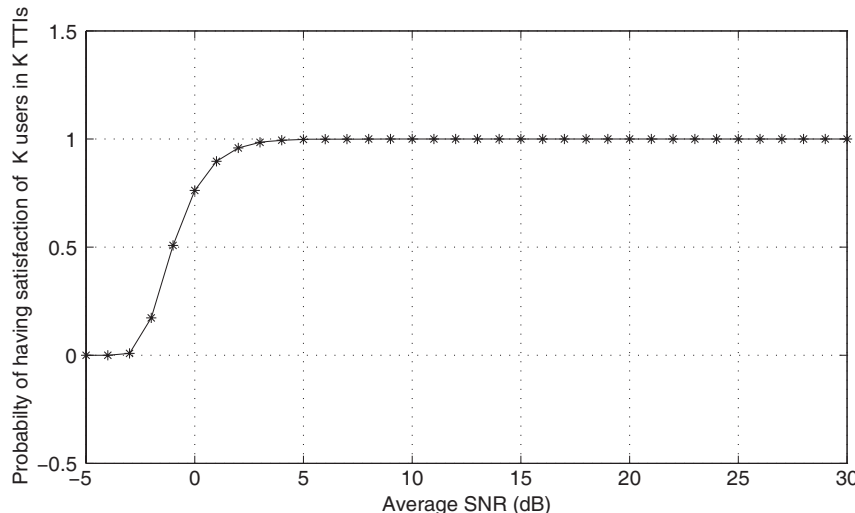

Fig. 8. Probability of having access of all the $K$ users in $K$ time slots versus the average SNR for the three schemes when $L_{T}=10, L=6, L_{c}=4$, $K=10, N=8$, and $B E R_{0}=0.001$.

conjunction with EEA to achieve better short term fairness of access between the i.i.d. users. In this case, the network scheduler removes the scheduled user in the next time slots until serving all the available users in the cell. It was shown that in good channel conditions, EEA guarantees for each user to have a another access to the channel after maximally $2 K-1$ time slots. It was also shown that this improvement in fairness is accompanied by a feedback load gain, but at the expense of a reduction in average spectral efficiency per time slot.

\section{REFERENCES}

[1] D. Gesbert and M.-S. Alouini, "Selective multi-user diversity," in Proc. IEEE International Symp. on Signal Processing and Information Technology (ISSPIT'03), Dresden, Germany, Dec. 2003, pp. 162-165.

[2] B. Holter, M.-S. Alouini, G. E. Øien, and H.-C. Yang, "Multiuser switched diversity transmission," in Proc. IEEE Veh. Technol. Conf. (VTC'04), vol. 3, Los Angeles, CA, USA, Sept. 2004, pp. 2038-2043.

[3] Y. A. Harthi, A. Tewfik, and M.-S. Alouini, "Multiuser diversity with quantized feedback," IEEE Trans. Wireless Commun., vol. 6, pp. 330337, Jan. 2007.

[4] M.-S. Alouini and A. J. Goldsmith, "Adaptive modulation over Nakagami fading channels," Kluwer J. Wireless Communications, vol. 13, pp. 119-143, May 2000.

[5] A. Goldsmith and S.-G. Chua, "Adaptive coded modulation for fading channels," IEEE Trans. Commun., vol. 46, pp. 595-602, May 1998.

[6] K. Hole, H. Holm, and G. E. Øien, "Adaptive multidimensional coded modulation over flat fading channels," IEEE J. Select. Areas Commun., vol. 18, pp. 1153-1158, July 2000.

[7] M.-S. Alouini and H.-C. Yang, "Minimum estimation and combining generalized selection combining (MEC-GSC)," in Proc. IEEE Int. Sym. Inform. Theory (ISIT'05), Adelaide, Australia, Sept. 2005, pp. 526-532.

[8] H.-C. Yang, "New results on ordered statistics and analysis of minimumselection generalized selection combining (GSC)," IEEE Trans. Wireless Commun., vol. 5, pp. 1876-1885, July 2006.

[9] H.-C. Yang and M.-S. Alouini, "MRC and GSC diversity combining with an output threshold," IEEE Trans. Veh. Technol., vol. 54, pp. 10811090, May 2005.

[10] M. K. Simon and M.-S. Alouini, Digital Communication over Generalized Fading Channels: A Unified Approch to Performance Analysis. New York, NY: John Wiley \& Sons, 2000.

[11] H.-C. Yang, N. Belhaj, and M.-S. Alouini, "Performance analysis of joint adaptive modulation and diversity combining over fading channels," IEEE Trans. Commun, vol. 55, pp. 520-528, Mar. 2007.

[12] S. Choi, M.-S. Alouini, K. A. Qaraqe, and H.-C. Yang, "Soft handover overhead reduction by RAKE reception with finger replacement," in Proc. IEEE International Conf. on Commun. Systems (ICCS'06), Singapore, Nov. 2006.

[13] K.-H. Park, Y.-C. Ko, and M.-S. Alouini, "Joint adaptive combining and multiuser down-link scheduling," IEEE Trans. Veh. Technol., vol. 57, pp. 2958-2968, Sept. 2008. 
[14] P. Viswanath, D. N. C. Tse, and R. Loria, "Opportunistic beamforming using dump antenna," IEEE Trans. Inform. Theory, vol. 6, pp. 12771294, June 2002.

[15] M. Johansson, "Diversity-enhanced equal access-considerable throughput gains with 1-bit feedback," in Proc. IEEE Workshop on Signal Processing Advances in Wireless Communications (SPAWC'04), Lisbon, Portugal, July 2004, pp. 6-10.

[16] Y. A. Harthi, A. Tewfik, and M.-S. Alouini, "Multiuser diversityenhanced equal access with quantized feedback in multicarrier OFDM systems," in Proc. IEEE Vehicular Technology Conference, (VTC'05Fall), Dallas, TX, Sept. 2005, pp. 568- 572.

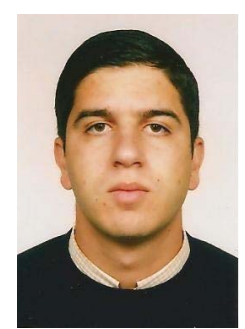

Slim Ben Halima was born in Sfax, Tunisia. He received the engineering Diploma from the Tunisia Polytechnic School, La Marsa, Tunisia, in 2007. $\mathrm{He}$ received the master degree in Digital Telecommunication Systems from Pierre et Marie Curie University, Paris, France, in 2008.

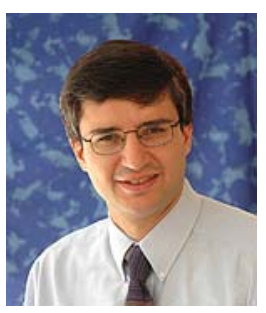

Mohamed-Slim Alouini (S'94, M'98, SM'03, F'09) was born in Tunis, Tunisia. He received the Ph.D. degree in electrical engineering from the California Institute of Technology (Caltech), Pasadena, CA, USA, in 1998. He was an Associate Professor with the department of Electrical and Computer Engineering of the University of Minnesota, Minneapolis, MN, USA. Since July 2005, he has been with the Electrical and Computer Engineering Program of the Texas A\&M University at Qatar, Education City, Doha, Qatar, where his current research interests include the design and performance analysis of wireless communication systems.

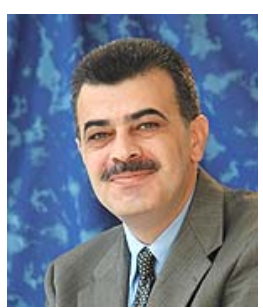

Khalid A. Qaraqe (M'97-S'00) was born in Bethlehem. Dr Qaraqe received the B.S. degree in EE from the University of Technology, Baghdad in 1986, with honors. He received the M.S. degree in EE from the University of Jordan, Jordan, in 1989, and he earned his Ph.D. degree in EE from Texas A\&M University, College Station, TX, in 1997. From 1989 to 2004 Dr Qaraqe has held a variety positions in many companies and he has over 12 years of experience in the telecommunication industry. Dr Qaraqe has worked for Qualcomm, Enad Design Systems, Cadence Design Systems/Tality Corporation, STC, SBC and Ericsson. He has worked on numerous GSM, CDMA, WCDMA projects and has experience in product development, design, deployments, testing and integration. Dr Qaraqe joined the department of Electrical Engineering of Texas A\&M University at Qatar, in July 2004, where he is now a senior associate professor.

Dr Qaraqe research interests include communication theory and its application to design and performance, analysis of cellular systems and indoor communication systems. Particular interests are in the development of WCDMA and broadband wireless communications and diversity techniques. 